\title{
BMJ Open EQ-VT protocol: one-size-fits-all? Challenges and innovative adaptations used in Egypt: a cross-sectional study
}

\author{
Sahar Al Shabasy, Maggie Abbassi, Samar Farid
}

To cite: Al Shabasy S, Abbassi M, Farid S. EQ-VT protocol: one-size-fits-all? Challenges and innovative adaptations used in Egypt: a cross-sectional study. BMJ Open 2021;11:e051727. doi:10.1136/ bmjopen-2021-051727

- Prepublication history for this paper is available online. To view these files, please visit the journal online (http://dx.doi org/10.1136/bmjopen-2021051727).

Received 30 March 2021 Accepted 30 November 2021

Check for updates

(C) Author(s) (or their employer(s)) 2021. Re-use permitted under CC BY-NC. No commercial re-use. See rights and permissions. Published by BMJ.

Department of Clinical Pharmacy, Cairo University Faculty of Pharmacy, Cairo, Egypt

Correspondence to Professor Samar Farid; samar.farid@pharma.cu.edu.eg

\section{ABSTRACT}

Objectives To present the challenges and adaptations done to the EuroQol Valuation Technology (EQ-VT) protocol to fit the Egyptian culture during the extensive pilot phase of the Egyptian EuroQol 5 Dimension five level (EQ-5D-5L) valuation study

Design This study was a cross-sectional, intervieweradministered face-to-face survey of representative Egyptians using the Arabic version of the EuroQol Group Valuation Technology (EQ-VT-2.1) and a country specific questionnaire pertaining to participants' demographics and opinions about health, life and death

Setting Participants were recruited from workplaces, university campuses, sporting clubs, shopping malls and other public areas from different Egyptian governorates representing all geographical areas of the country. Participants A total of 1378 participants were interviewed from July 2019 to March 2020 by 12 interviewers to select a representative sample in terms of: geographical distribution, age and gender, of which 75 participants did not complete the interview, 298 interviews were pilot and 1005 interviews were real of which 974 interviews were used for the valuation study. Two participants did not complete the country-specific questionnaire but completed the valuation protocol; therefore, 1301 interviews were included in the final analysis of country specific questions.

Results Some modifications were applied to the protocol. The 'wheelchair example' was modified to 'migraine example' since most of the participants in the pilot interviews considered being in a wheelchair 'worse than dead'. There was some ambiguity in the Egyptian translated version for the EQ-5D-5L between levels 4 and 5 of the pain and depression dimensions. This was overcome by using colour coding to express the different levels of severity. A pictorial representation for the EQ-5D$5 \mathrm{~L}$ health states was used to interview illiterate and less educated participants.

Conclusion In the Egyptian valuation study, the modifications made to the EQ-VT protocol made it feasible and culturally acceptable to the Egyptian participants.

\section{INTRODUCTION}

The EuroQol 5 Dimension (EQ-5D) is a health-related quality of life (HRQOL) questionnaire developed by the EuroQol group. It is widely used by health technology assessment agencies and recommended by most of

\section{Strengths and limitations of this study}

- Some adaptations were applied to the EuroQol Valuation Technology (EQ-VT) protocol in the initial health example used to make it feasible and culturally acceptable to the Egyptian participants.

- Using a pictorial representation and colour coding of the EuroQol 5 Dimension five level (EQ-5D-5L) was very helpful in describing the provided health states and levels of severity, particularly with illiterates and less educated participants.

- The adaptation of the EQ-VT protocol and the interview process has resulted in the success of using such valuation techniques on Egyptian participants with different social, religious and educational backgrounds.

- This study may encourage other Muslim and Arabic speaking countries, and possibly other different cultures, to follow Egypt's steps and to develop their own value set.

- The tools used for the pictorial representation and the colour coding of the EQ-5D-5L health states were not fully validated.

the pharmacoeconomic guidelines. ${ }^{1-3}$ There are two versions of the instrument for adults; the three level EQ-5D (EQ-5D-3L) which is available in more than 170 languages and the five level EQ-5D (EQ-5D-5L) which is available in more than 130 languages. ${ }^{4}$ The $5 \mathrm{~L}$ version produces a more accurate measurement of patient health than the $3 \mathrm{~L}$ version. ${ }^{5}$ The EuroQol group developed a standardised valuation protocol for the EQ-5D-5L valuation studies to enhance comparability of valuation studies and to investigate the effect of cultural issues and other factors on health utility values among countries. ${ }^{6}$

The EuroQol Valuation Technology (EQVT) includes two valuation techniques: the composite time trade off (cTTO) and discrete choice experiment (DCE). In cTTO, all the health states are traded against time to be better than dead (BTD) or worse than dead (WTD). Since this could be challenging to understand, the valuation protocol includes 
warm up cTTO tasks using the health state 'being in a wheel chair' as an example to explain the BTD state, followed by a task where the participant is asked to imagine a health state that is much worse than being in a wheelchair to explain the WTD element of the cTTO and illustrate the full scale available for tradeoff. This is followed by three practice states to familiarise the participants with the different severity levels of the health states and assess their comprehension and acceptability of the task. ${ }^{67}$ Whereas in DCE tasks, participants are offered a pair of health states with different severity levels and asked to choose one over the other. ${ }^{7}$

Considerable variations in health valuation have been noted across countries due to social, cultural and religious factors in addition to the used elicitation technique and analysis. ${ }^{8-10}$ For example, the concept of worse-thandead might not be fully acceptable in some cultures. ${ }^{11}$ Furthermore, the use of cTTO and DCE in the EQ-5D valuation studies require that the participants are able to read and understand the tasks, thus illiterate participants may not be able to complete text-based TTO and DCE tasks. Therefore, in countries where illiteracy is high, the use of the valuation techniques could be challenging.

An investigational study performed in the United Arab Emirates ${ }^{12}$ provided several insights into the feasibility and cultural appropriateness of using the EuroQol Group's standardised valuation protocol and recommended future studies to describe which specific elements of the protocol should be adapted.

The aim of this article is to present the challenges and adaptations done to the EQ-VT protocol to fit the Egyptian culture during the pilot phase of the Egyptian valuation study for the EQ-5D-5L. ${ }^{13}$

\section{METHODS}

\section{Study design}

This was a face-to-face cross-sectional study where interviewers administered the survey to the general Egyptian population using the EQ-VT-2.1 protocol and added a country-specific questionnaire designed for the Egyptian valuation study.

\section{Study preparation}

The principal investigators (PIs) received a 2-day training in the EuroQol headquarters. An Arabic version of the EQ-VT-2.1 was prepared for Egypt. Translation and proofreading of all the specific elements of the protocol was carefully done. Since the Arabic language is written from right to left, there were several issues in the earlier versions of the software. The PIs did more than 20 test interviews to test the software and feedback reports were sent to the EQ-VT technical support team for modification and correction before the final version was approved.

The training material used for the interviewer training and the Arabic version of the interviewers' instructions were the standard ones provided by The EuroQol Group. Since, in Egypt, the spoken Arabic language is different from the written language, the interviewers' instructions were rephrased as a unified script using the spoken Arabic language to be easily understood by participants. A country specific questionnaire was prepared by the research team. The participants were asked about their education, geographical area within Egypt, residence, religious beliefs, marital and employment status, health insurance availability, current illness and beliefs about life and death. In addition, participants were asked if the valuation tasks caused any conflicts with their religious beliefs, their concern about serious illness and were asked to rank the five dimensions from the most important to the least important from their point of view.

The PIs started initial pilot interviews $(\mathrm{N}=25)$ on the interviewers-to-be (before the interviewer training) and other academic researchers to explore any linguistic or semantic difficulties and to gain some experience in the valuation tasks before training the interviewers.

\section{Interviewers' training}

The interviewer team included 12 interviewers (11 females and 1 male), all were teaching assistants at the Clinical Pharmacy Department, Faculty of Pharmacy, Cairo University. The training was performed in four stages: during the first stage all the interviewers did the valuation interview as participants with one of the PIs, in the second stage, the interviewers received intensive 1-day training by the PIs, the training followed the standard procedure recommended by the EuroQol. The training materials included four modules: (1) goals and benefits of the study and introduction of related concepts such as valuing HRQOL, economic evaluation of health technologies and the EQ-5D-5L as a generic questionnaire used to value health states, (2) contents of the valuation interview, (3) interviewer instructions and demo interview and (4) quality control (QC) process. In the third stage, the interviewers were asked to study the interviewer's instructions and do at least two test interviews to become familiar with the contents of the valuation protocol and the software. In the fourth and last stage, each interviewer did one test interview with one of the PIs in the role of a participant and another two pilot interviews while being observed by of one of the investigators for advice and feedback to establish which element(s) of the valuation interview needed improvement and possibly, retraining. The initial pilot interviews were done on family and friends who were highly educated participants living in urban areas in Cairo to gain experience on the valuation tasks. Throughout the study, interviewers asked participants to read the health states out loud to ensure that they were able to read and understand the tasks. Interviewers were also asked to provide written feedback after each interview to record and predict possible challenges during the interview process. Follow-up of the interviewers was performed by the study PIs through periodical online and face to face meetings during the whole data collection process. 


\section{Study population and sampling method}

The Egyptian participants who agreed to give written informed consent, aged 18 years or more and were able to understand the valuation tasks in the familiarisation session of the 'wheelchair example' and the practice health states were included in the study. Participants were recruited using multistratified quota sampling based on the Egyptian official statistics updated in March $2019^{14}$ to select a representative sample in terms of: geographical distribution, age and gender. In accordance with the EQ-VT protocol for EQ-5D-5L valuation studies, a sample size of 1000 is recommended. ${ }^{612}{ }^{15}$ Potential participants were approached in workplaces, university campuses, sporting clubs, shopping malls and other public areas. All required permissions were obtained from different local official and non-official authorities before travelling to recruitment sites to facilitate data collection. In most cases, the interviews took place alongside recruitment. Interviewers were instructed to do the interviews with participants in quiet areas and not in presence of other participants to avoid any distraction. Participants did not receive any incentives.

\section{QC process and pilot phase}

The EuroQol group developed a QC process for the EQ-5D-5L valuation studies where it provides a number of criteria to evaluate interviewers performance and to improve the quality of the collected data. ${ }^{16}$ An extensive pilot phase $(\mathrm{n}=298$ interviews $)$ took place from July to October 2019.The objectives of the pilot phase were to test the feasibility and cultural appropriateness of the EQ-VT protocol on the Egyptian participants and to ensure the validity of the collected valuation data by standardising the interviewers' performance. The interviewers' performance and protocol compliance were monitored through the QC tool. The sample size of the pilot phase was not predetermined where the interviewers continued to perform pilot interviews till their performance and the quality of the collected data was acceptable to the researchers and EQ-VT support team.

Some challenges were encountered during the pilot phase related to obtaining written informed consent, acceptability of the wheel chair example, the Arabic translation of the levels of severity of the health states and interviewing illiterate participants. Periodical meetings were arranged between the Egyptian team and the EuroQol support team every week during the pilot phase (every 5 interviews per interviewer) and every 2 weeks in the actual data collection phase (every 10 interviews per interviewer) for the discussion of the QC reports, challenges and suggested adaptations used during the pilot phase and the whole data collection process. Any changes made to overcome challenges were adopted early on in the pilot phase.

\section{Patient and public involvement}

No patients or members of public were invited to participate in the study design, conduct, reporting or dissemination plans for this research.

\section{Data analysis}

Descriptive statistics were produced using IBM SPSS Statistics for Windows, V.22.0 (IBM Corp.) to describe the sample characteristics and the responses to the country specific questions (frequencies and percentages for discrete variables, mean and SD for continuous variables) and were compared using one sample z-test for a population proportion and $\chi^{2}$ goodness-of-fit test, respectively.

\section{RESULTS}

\section{Study sample characteristics}

A total of 1378 interviews were conducted from July 2019 to March 2020, of which 75 interviews were incomplete, 2 participants did not complete the country-specific questionnaire but completed the valuation protocol. The reason of the incomplete interviews was as follows: religious beliefs $(n=30)$, length of the interview $(n=17)$, the participants could not understand the valuation tasks $(n=18)$, technical problems $(n=5)$ and other non-specified reasons $(n=5)$. For the valuation study, only 974 interviews of the conducted interviews were considered. The rest were part of the pilot phase. However, since the countryspecific questionnaire data are still valid and not directly linked to the valuation tasks, 1301 responses were analysed.

The study sample characteristics in comparison with the Egyptian general population ${ }^{14}{ }^{17}$ are shown in table 1. Compared with national statistics there were some differences in the distribution of the background variables with respect to age, education, geographical distribution, marital status and health insurance. Despite these differences, the demographic characteristics still produced the required diversity.

\section{Country-specific questionnaire}

The majority of participants (80.9\%) stated that living a healthy life regardless of its length was more important than living a long life regardless of its quality, $92.4 \%$ of the participants stated that the valuation tasks did not cause any conflicts with their religious beliefs and $87.2 \%$ stated that their religious beliefs did not influence their response to the valuation tasks (figure 1A). In case of serious illness, the two major concerns of participants were: becoming a burden on family and friends $(36.9 \%)$ and not being able to take care of their families (24.3\%). About $45 \%$ of the participants stated that their approach to illness depends on the health condition (figure 1B). Out of our sample, $31.8 \%$ stated that they were diagnosed with chronic health conditions, the majority of which $(73.9 \%)$ suffered from only one chronic health condition (table 1). Of the five EQ-5D dimensions, mobility was ranked as the most important dimension by $41.2 \%$ of participants followed by anxiety depression $(15.7 \%)$, usual activities $(14.7 \%)$, selfcare $(14.6 \%)$ and pain/discomfort (13.8\%).

\section{Challenges in the interview process Informed consent}

During the pilot phase, it was easy to take written informed consents as most of the interviews were performed on 
Table 1 Participant characteristics answering the country specific questionnaire compared with the Egyptian population

\section{Characteristics}

Full sample ( $n=1301), N(\%)$

General population* (\%)

$P$ value

Sext

Male

Female

Age (years) $\dagger$ mean \pm SD

Range

Age group (years) $\uparrow$

$\begin{array}{ll}18-34 & 680(52.2) \\ 35-54 & 491(37.7) \\ \geq 55 & 132(10.1)\end{array}$

Geographical region

\begin{tabular}{|c|c|c|c|}
\hline Greater Cairo & $511(39.3)$ & 25.1 & 0 \\
\hline Alexandria & $123(9.5)$ & 12.4 & 0.0015 \\
\hline Delta & $229(17.6)$ & 21.7 & 0.0003 \\
\hline Suez Canal & $123(9.5)$ & 11.2 & 0.0519 \\
\hline North upper Egypt & $144(11.1)$ & 12.9 & 0.0528 \\
\hline Asyut & $45(3.5)$ & 4.9 & 0.0193 \\
\hline South upper Egypt & $126(9.7)$ & 11.8 & 0.0189 \\
\hline \multicolumn{4}{|l|}{ Residence } \\
\hline Urban & $934(71.8)$ & 42.2 & 0 \\
\hline Rural & 367 (28.2) & 57.8 & 0 \\
\hline \multicolumn{4}{|l|}{ Education level } \\
\hline Illiterate & $116(8.9)$ & 25.8 & 0 \\
\hline Below intermediateł & $311(23.9)$ & 29 & $<0.0001$ \\
\hline Intermediate§ & $511(39.3)$ & 29.1 & $<0.0001$ \\
\hline University degree and above & $363(27.9)$ & 15.5 & 0 \\
\hline \multicolumn{4}{|l|}{ Employment status } \\
\hline Employed & $950(73)$ & 74.4 & 0.2472 \\
\hline Unemployed/retired/students & $351(27)$ & 25.6 & 0.2472 \\
\hline \multicolumn{4}{|l|}{ Marital status } \\
\hline Married & $740(56.8)$ & 68 & 0 \\
\hline Single/divorced/widowed & $561(43.2)$ & 32 & 0 \\
\hline \multicolumn{4}{|l|}{ Religious beliefs } \\
\hline Muslims & $1241(95.4)$ & 94.99 & 0.4123 \\
\hline Christians & $60(4.6)$ & $5.1 \rrbracket$ & 0.4123 \\
\hline \multicolumn{4}{|l|}{ Health insurance } \\
\hline Covered (full or partial) & $786(60.4)$ & 54.7 & $<0.0001$ \\
\hline No coverage & $515(39.6)$ & 45.3 & $<0.0001$ \\
\hline \multicolumn{4}{|c|}{ Diagnosed with chronic health condition } \\
\hline None & 887 (68.2) & & \\
\hline 1 & $306(23.5)$ & & \\
\hline 2 & $83(6.4)$ & & \\
\hline$\geq 3$ & $25(1.9)$ & & \\
\hline \multicolumn{4}{|l|}{ Experience with serious illness $†$} \\
\hline In self & $230(17.7)$ & & \\
\hline In family & $726(55.7)$ & & \\
\hline
\end{tabular}

Continued 
Table 1 Continued

\begin{tabular}{clll}
\hline Characteristics & Full sample $(\mathbf{n}=1301), \mathbf{N}(\%)$ & General population* $(\%)$ & $\mathbf{P}$ value \\
\hline In caring for others & $509(39.1)$ & \\
\hline
\end{tabular}

*Data estimated from the Egyptian Central Agency for Public Mobilisation and Statistics (CAPMAS), March 2019. ${ }^{14}$

†Sample size for the sample is $n=1303 p<0.05$ (based on 1-sample $z$ test for a population proportion).

Below intermediate: below high school level.

§Intermediate: high school level or 2 years institute.

qData obtained from: Mohamoud et al. ${ }^{17}$

family and friends, but during actual data collection, most participants feared to sign a consent form. This was solved by using a collective informed consent form where the study objectives were mentioned then more than one participant could sign in a table underneath. All participants were informed about their freedom of refusal and that all research data was anonymous and confidential.

\section{Wheelchair example}

The first task in the cTTO training set is to ask the participants to imagine that they are in a wheelchair, which is expected to elicit a better-than-dead response. However, during the early pilot interviews, 12 participants out of 18 considered being in a wheelchair was worse than being dead. This presented a problem in the flow of the training tasks which were designed to show the BTD elements first followed by the WTD ones thus revealing the full scale of TTO. Therefore, the 'wheelchair example' needed to be changed to a health state that was easily understood, gave no opportunity for ambiguity and was generally perceived as BTD by most. 'Migraine example' was chosen to replace the wheelchair example. This decision, taken together with the EuroQol support team, had the positive effect of ensuring consistency with other valuation studies since the BTD state was revealed and explained before the WTD state. Therefore, the structure of the familiarisation tasks remained unchanged with the application of the same QC criteria.

\section{Colour coding of the severity levels}

There was some ambiguity in the Egyptian version of the EQ-5D-5L, for the fourth and fifth levels in the translation of 'severe' and 'extreme'. This issue was raised during the phase of proofreading and piloting where the wording used in the Arabic version for Egypt confused the participants in the pain/discomfort and anxiety/depression

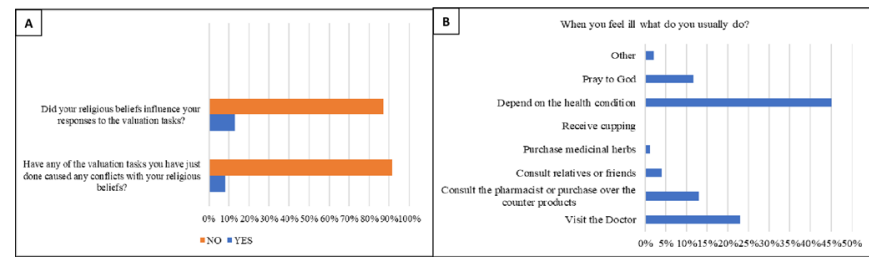

Figure 1 Response of participants (percentage) about conflicts with religious beliefs $(A)$, and concerns about illness (B) $(N=1301)$ ( $p$ value $=0.000$ using the $\chi^{2}$ goodness of fit to test the differences between percentages). dimensions. The Arabic word used to describe level 4 in these two dimensions could mean sharp, pointed, severe, intense or acute and when used to describe a particular health state, the participants were unable to determine if the level of severity was 4 or 5 . This issue was overcome by using a hard copy of the EQ-5D-5L with colour codes to define the five levels of severity (figure 2A). The colours green, yellow, orange, red and deep crimson were used to represent the five levels of severity. The colours were adapted from the traffic light system which was familiar to the participants. All interviewers were instructed to use the colour codes for all participants at the beginning of the interview for the self-reported health of the EQ-5D-5L questionnaire and allow the participants to familiarise themselves with it and be able to refer to it throughout the interview. This issue was also raised with the EuroQol Version Management Committee and a decision was made to change the wording used in the Arabic version of the EQ-5D-5L according to our recommendation.

Pictorial representation of EQ-5D-5L health states for illiterate and less educated participants

In Egypt, the percentage of illiterate individuals was reported to be $25.8 \%$ in $2019 .{ }^{14}$ The EQ-VT protocol is designed for literate individuals; however, the investigators decided that illiterates should not be neglected in the Egyptian valuation study. A novel tool was created by the Egyptian team which included pictorial representation of the EQ-5D-5L health states. Graphics were used to describe the five dimensions and coloured cards were

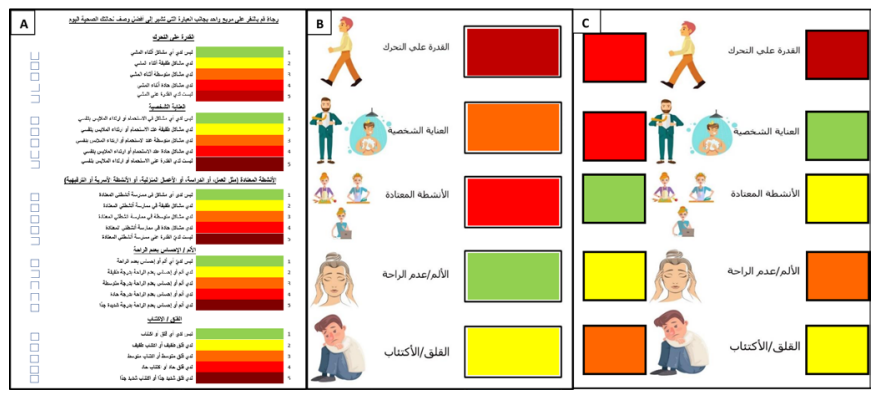

Figure 2 The colour-coded EuroQol 5 Dimension five level (EQ-5D-5L) (A) and the pictorial representation of the EQ-5D health states for composite time trade off task (health state 53412) (B) and for discrete choice experiment (DCE) task (health states: 44123 left, 51232 right) (C). Green=level 1 (no problems), yellow=level 2 (mild), orange=level 3 (moderate), red=level 4 (severe), crimson=level 5 (extreme/not able). 
used to describe the five levels for both cTTO and DCE tasks (figure $2 \mathrm{~B}$ and $\mathrm{C}$ ). The pictorial representation and the colours were first evaluated by the Egyptian team together with the EuroQol group support team. Furthermore, this tool was tested on three illiterate participants asking them about their feedback before using them in actual interviews. The interviewers were then trained on how to use the coloured cards to interview illiterate and poorly educated participants who were not able to properly read and understand the tasks. Literacy competency was judged by the interviewers, where participants who could not read the self-reported health on the EQ-5D-5L descriptive system at the beginning of the interview, even if they stated that they received some education, were considered illiterate. Interviewers were instructed to read the health states out loud twice to the participants while placing the corresponding-coloured cards in front of the graphics representing each of the five dimensions. This tool was used for 116 participants.

\section{Cultural and religious beliefs}

It was uncomfortable for some participants to imagine the health states on themselves and think about illness and death. The DCE generally seemed more acceptable than the TTO as trading between life and death was difficult for some participants due to their religious beliefs. Some participants refused the concept of the WTD and some participants refused to complete the interview for the same reason. This was overcome early in the pilot phase by giving detailed explanation of the study objectives and reassuring the participants that the valuation tasks were not intended to cause any conflict with their spiritual or religious beliefs in any way and that life and death are in God's hand. Furthermore, three participants refused to trade any life years, even for the worst health state, and said they will accept illness with patience while other participants used to trade all life years if they found extreme or severe problems in the displayed health states for fear of becoming a burden on family and friends.

\section{Other challenges reported by the interviewers}

Other issues were reported by the interviewers during the initial phase of pilot interviews. Some participants reported that some health states were illogical and difficult to imagine, became tired and did not want to review the feedback module, refused to enter the WTD element in the wheel chair example or had inconsistent responses in the cTTO and DCE tasks. Some clustering of values and some bias was observed while interviewing members of close family and friends. The following recommendations were applied: The team agreed to provide the participants with examples demonstrating seemingly illogical health states that would help thought processes during the valuation tasks. The interviewers were instructed to spend more time in the feedback module and revise each health state with the participants to ensure that their choices reflected their real preferences. For the participants who refused to use the lead time in the migraine example, the interviewers were instructed to show all the screen elements in the WTD part and then reset the example to take the participants' preferences and to ask the participants for the rationale of their choices if their answers were inconsistent. It was advised not to include family and friends in actual valuation interviews to avoid any emotional influence.

\section{DISCUSSION}

This study reports cultural issues and challenges that arose during the use of the preference methods in Egypt. Moreover, it explores the methodological adaptation in the EQ-VT protocol and the interview process to make it feasible and culturally acceptable to Egyptian participants.

The EuroQol group designed the standardised EQ-VT protocol for the EQ-5D-5L valuation studies so the different national tariffs can be easily compared across countries. However, some adaptations may be required in the protocol and the interview process to be easily administered in countries with religious, economic, social and cultural backgrounds different than those where the protocol was developed.

In Egypt and many other developing countries, independent living in a wheelchair is challenging due to unequipped public transportation and lack of wheelchairfriendly infrastructure like ramps and elevators in most buildings, which may have prompted most participants to decide that living in a wheelchair was a fate worse than death. Mobility was chosen as the most important dimension by more than $40 \%$ of participants. Thus, the use of the 'wheelchair example' as the initial cTTO example was not acceptable for most of the participants which necessitated changing it to the 'migraine example'. To the best of our knowledge this is the first adaptation to the EQ-VT protocol in terms of the initial health cTTO example compared with other published valuation studies. ${ }^{18}$ In Vietnam the third person approach was used in the cTTO task due to the sensitivity of talking about illness and death in the Vietnamese culture. ${ }^{11}$

It was challenging to interview illiterate and less educated participants as the EQ-VT protocol is designed for literate participants. This necessitated the development of visual aids with pictorial representations and coloured cards to represent the different dimensions of health states and levels of severity for both the cTTO and DCE tasks. In the Moroccan EQ-5D-3L valuation study graphical representations for all the TTO and DCE health states included in the design were created and used for illiterate participants. ${ }^{19}$ while in Vietnam, visual aids were used for elderly participants for the DCE based tasks only. ${ }^{11}$ In addition, colour coding to define severity levels has been previously used ${ }^{2021}$

The health preferences of Muslim countries might be influenced by religious beliefs where some participants reported that they were not allowed to "choose" as everything is in God's hand and they believed strongly in fate and destiny. This is in line with other published 
studies. ${ }^{122-25}$ However, in Egypt, this turned out not to be a major concern. Almost all participants traded many life years if there were any severe or extreme problems in any of the dimensions of the shown health states and indicated that being a burden on family and friends was their major concern as stated in the country specific questions. The percentage of WTD responses in Egypt was relatively high $(40.9 \%)$ when compared with other countries. ${ }^{26} 27$ Again, this is in line with the other collectivistic cultures such as Indonesia. ${ }^{26}$ In Egypt, the majority of participants stated that the valuation tasks did not cause any conflicts with their religious beliefs $(92.4 \%)$ and that their religious beliefs did not influence their preferences (87.2\%). This, in part, was achieved by the detailed explanation of the study aims and benefits given to all participants and by reassuring them that the valuation tasks were designed to obtain their preference and opinion regarding some health states and not intended to cause conflict with their religious beliefs in any way. Participants were also assured that the study results will be used for resource allocation decisions and the improvement of the healthcare system in Egypt.

The interviewers training was done on four stages to ensure the quality of the interview process. Conducting the interviews on the interviewers before the training had a positive impact on the training session and the subsequent interview process.

Although this study provides important insights on the challenges and adaptations of the EQ-VT protocol to fit the Egyptian culture, some limitations are noted. First there were some deviations in the background sample characteristics compared with the general population as the sample used in this study included the pilot interviews which were conducted, for convenience, on educated participants living in urban areas in Cairo. Second, the tools used for the pictorial representation and the colour coding of the EQ-5D-5L health states were not fully validated. However, this study may encourage other Muslim and Arabic speaking countries to follow Egypt's steps and to develop their own value set when provided with some tried and tested culture-appropriate adaptations. It is also worthy to mention that some of the adaptations, like the combined informed consent, can have wider application to reduce the level of apprehension of sceptical participants without violating ethical requirements and participants' rights.

\section{Recommendations}

Adding visual aids describing the health states and levels of severity to be displayed on the screen of the EQ-VT software will enable its standardised use with participants suffering from reading problems and, possibly, some cognitive difficulties. Moreover, using collective informed consent and using migraine as the initial health example might be beneficial for valuation studies in some countries with cultural and social backgrounds comparable to Egypt.

\section{CONCLUSIONS}

In the Egyptian valuation study, the EQ-VT protocol 2.1 needed modification in terms of the initial health example used. Using a pictorial representation and colour coding was helpful in defining the levels of severity for the provided health states, particularly with participants unable to read. The EQ-VT protocol may not fit every country/culture/language around the world, but it seems flexible enough to be resized while keeping the same structure and QC process.

Acknowledgements We would like to thank the EuroQol support team: Elly Stolk, Fatima Al Sayah, Aureliano Paolo Finch and Arnd Jan Prause for their guidance and support during study preparation, data collection and quality control process. We are grateful for the outstanding effort of the interviewers. Finally, we thank the study participants for taking part in this study.

Contributors SAS participated in the study preparation, data collection, performed data quality control reports, analysis, interpretation of results and prepared the draft manuscript. MA and SF participated in the study preparation, proof reading of the translated version, follow-up of the data collection, quality control process, interpretation of results and reviewed the final manuscript. SF is the guarantor. All authors approved the final version for publication.

Funding This project received financial support from Bournemouth University and the EuroQol foundation, The Netherlands (project ID: 20180130). The funding agreement ensured the authors' independence in designing, writing and publishing the study results.

\section{Competing interests None declared.}

Patient and public involvement Patients and/or the public were not involved in the design, or conduct, or reporting, or dissemination plans of this research.

\section{Patient consent for publication Not applicable.}

Ethics approval The study proposal was approved by the EuroQol group. The study received ethical approval from the Ethics Committee of Faculty of Pharmacy Cairo University ((Ethics approval ID: CL 2253). and was conducted in accordance with the Declaration of Helsinki. Written informed consent was obtained from all participants included in the study.

Provenance and peer review Not commissioned; externally peer reviewed.

Data availability statement Data are available upon reasonable request. The datasets generated and analysed during the current study are available from the corresponding author upon reasonable request.

Open access This is an open access article distributed in accordance with the Creative Commons Attribution Non Commercial (CC BY-NC 4.0) license, which permits others to distribute, remix, adapt, build upon this work non-commercially, and license their derivative works on different terms, provided the original work is properly cited, appropriate credit is given, any changes made indicated, and the use is non-commercial. See: http://creativecommons.org/licenses/by-nc/4.0/.

ORCID iD

Samar Farid http://orcid.org/0000-0002-6048-847X

\section{REFERENCES}

1 Kennedy-Martin M, Slaap B, Herdman M, et al. Which multi-attribute utility instruments are recommended for use in cost-utility analysis? A review of national health technology assessment $(\mathrm{HTa})$ guidelines. Eur $J$ Health Econ 2020;21:1245-57.

2 Brauer CA, Rosen AB, Greenberg D, et al. Trends in the measurement of health utilities in published cost-utility analyses. Value Health 2006;9:213-8.

3 Prosser L, Wittenberg E, eds. Trends in utility elicitation methods: is there still a role for direct elicitation? In: 32nd Annual Meeting of. Toronto: Society for Medical Decision Making, 2010.

4 The EQ-5D instruments. Available: https://euroqol.org/ [Accessed 9 Oct 2020].

5 Janssen MF, Pickard AS, Golicki D, et al. Measurement properties of the EQ-5D-5L compared to the EQ-5D-3L across eight patient groups: a multi-country study. Qual Life Res 2013;22:1717-27. 
6 Oppe M, Devlin NJ, van Hout B, et al. A program of methodological research to arrive at the new international EQ-5D-5L valuation protocol. Value Health 2014;17:445-53.

7 Stolk E, Ludwig K, Rand K, et al. Overview, update, and lessons learned from the International EQ-5D-5L valuation work: version 2 of the EQ-5D-5L valuation protocol. Value Health 2019;22:23-30.

8 Knies S, Evers SMAA, Candel MJJM, et al. Utilities of the EQ-5D. Pharmacoeconomics 2009;27:767-79.

9 Kelleher D, Barry L, Hobbins A, et al. Examining the transnational health preferences of a group of eastern European migrants relative to a European host population using the EQ-5D-5L. Soc Sci Med 2020;246:112801.

10 Elbarazi I, Devlin NJ, Katsaiti M-S, et al. The effect of religion on the perception of health states among adults in the United Arab Emirates: a qualitative study. BMJ Open 2017;7:e016969.

11 Mai VQ, Sun S, Minh HV, et al. An EQ-5D-5L value set for Vietnam. Qual Life Res 2020;29:1923-33.

12 Papadimitropoulos EA, Elbarazi I, Blair I, et al. An investigation of the feasibility and cultural appropriateness of stated preference methods to generate health state values in the United Arab Emirates. Value Health Reg Issues 2015;7:34-41.

13 Al Shabasy S, Abbassi M, Finch A, et al. The EQ-5D-5L valuation study in Egypt. Pharmacoeconomics 2021;37:549-61.

14 Central agency for public mobilization and statistics - CAPMAS, 2019. Available: http://www.capmas.gov.eg/Pages/StaticPages. aspx?page id=5035 [Accessed 9 May 2019].

15 M Versteegh M, M Vermeulen K, M A A Evers S, et al. Dutch tariff for the five-level version of EQ-5D. Value Health 2016;19:343-52.

16 Ramos-Goñi JM, Oppe M, Slaap B, et al. Quality control process for EQ-5D-5L valuation studies. Value Health 2017;20:466-73.

17 Mohamoud YA, Cuadros DF, Abu-Raddad LJ. Characterizing the Copts in Egypt: demographic, socioeconomic and health indicators. Q Sci Connect 2013
18 EQ-5D-5L | valuation: standard value sets. Available: https://euroqol. org/eq-5d-instruments/eq-5d-5l-about/valuation-standard-valuesets/ [Accessed 20 Nov 2020].

19 Al Sayah F, Kooli A, Rouahi Y, et al. PNS324 A NOVEL APPROACH OF USING GRAPHICAL REPRESENTATION OF EQ-5D-3L DIMENSIONS, LEVELS, AND HEALTH STATES FOR ILLITERATE RESPONDENTS IN VALUATION STUDIES - AN EXAMPLE FROM MOROCCO. Value Health 2019;22:S819.

20 Jonker MF, Donkers B, de Bekker-Grob EW, et al. Effect of level overlap and color coding on attribute non-attendance in discrete choice experiments. Value Health 2018;21:767-71.

21 Jonker MF, Donkers B, de Bekker-Grob EW, et al. Advocating a paradigm shift in health-state valuations: the estimation of timepreference corrected QALY tariffs. Value Health 2018;21:993-1001.

22 Goudarzi R, Sari AA, Zeraati $\mathrm{H}$, et al. Valuation of quality weights for EuroQol 5-Dimensional health states with the time tradeoff method in the capital of Iran. Value Health Reg Issues 2019;18:170-5.

23 Yusof FAM, Goh A, Azmi S. Estimating an EQ-5D value set for Malaysia using time trade-off and visual analogue scale methods. Value Health 2012;15:S85-90.

24 Purba FD, Hunfeld JAM, Iskandarsyah A, et al. Employing quality control and feedback to the $E Q-5 D-5 L$ valuation protocol to improve the quality of data collection. Qual Life Res 2017;26:1197-208.

25 Wee H-L, Li S-C, Xie F, et al. Validity, feasibility and acceptability of time trade-off and standard gamble assessments in health valuation studies: a study in a multiethnic Asian population in Singapore. Value Health 2008;11 Suppl 1:S3-10.

26 Purba FD, Hunfeld JAM, Iskandarsyah A, et al. The Indonesian EQ5D-5L value set. Pharmacoeconomics 2017;35:1153-65.

27 Lin H-W, Li C-I, Lin F-J, et al. Valuation of the EQ-5D-5L in Taiwan. PLoS One 2018;13:e0209344. 